\title{
Biochemical Characterization of Serratia lique- faciens (Grimes and Hennerty) Bascomb et al. (Formerly Enterobacter liquefaciens) and Serratia rubidaea (Stapp) comb. nov. and Designation of Type and Neotype Strains
}

\author{
WILLIAM H. EWING, BETTY R. DAVIS, MARY A. FIFE, and ERWIN F. LESSEL \\ Center for Disease Control, Atlanta, Georgia 30333, and American Type Culture Collection, \\ Rockville, Maryland 20852
}

\begin{abstract}
A study was made of the biochemical reactions of 109 strains of Enterobacter liquefaciens (Grimes and Hennerty) Ewing and 49 isolants resembling Bacterium rubidaeum Stapp. The results supported the recent transfer of E. liquefaciens to the genus Serratia Bizio by Bascomb et al. and indicated that Bacterium rubidaeum Stapp should also be transferred to Serratia as Serratia rubidaea (Stapp) comb. nov., the previous use of the name Serratia rubidaea by Stapp not having constituted valid publication of this name. According to proposals made, three species are recognized in the genus Serratia: $S$. marcescens, $S$. liquefaciens, and $S$. rubidaea. Means for differentiating the three species are provided. Strains 1284-57 (= ATCC 27592) and 2199-72 (= ATCC 27593) are proposed as the type and neotype strains, respectively, of $S$. liquefaciens and $S$. rubidaea.
\end{abstract}

The microorganism orginally named Aerobacter liquefaciens by Grimes and Hennerty (17) was placed in the genus Enterobacter Hormaeche and Edwards by Ewing (6) in 1963. However, it has been known for some time that close biochemical affinities exist between $E_{n}$ terobacter liquefaciens (Grimes and Hennerty) Ewing and Serratia marcescens Bizio, the type species of Serratia Bizio $(5,8,10,11,12,14)$. Additional evidence of the relationship of these two microorganisms has been based upon studies with bacteriocins (18), production of nucleoside phosphotransferase (4), antibiotic sensitivities (15), analyses of biochemical data $(1,21)$, and degree of reassociation of deoxyribonucleic acids (3): In 1971, Bascomb et al. (1) transferred $E$. liquefaciens to the genus Serratia.

In this publication the results obtained from an examination of strains of $E$. liquefaciens and $S$. marcescens and isolates that resemble $\mathrm{Bac}$ terium rubidaeum Stapp $(2,22)$ are compared. The observed biochemical relationships of these microorganisms are reported, means are pro- vided for their differentiation, and proposals are made regarding their taxonomy and nomenclature. As stated elsewhere (5), the senior author has never been averse to reclassification of $E$. liquefaciens as a species of Serratia. A formal proposal to this effect would have been made earlier except that related problems, such as the taxonomic position of B. rubidaeum, required further investigation and clarification.

\section{MATERIALS AND METHODS}

Bacterial strains. Between January 1, 1957, and June 30, 1972, 109 strains of Enterobacter liquefaciens (Serratia liquefaciens hereafter) were received in the Enteric Bacteriology Laboratories, Laboratory Division, of the Center for Disease Control (CDC). Of these, 68 were mentioned in a previous publication (14). Six of the isolants were supplied by culture collections such as the American Type Culture Collection (ATCC). Two of the six (1284-57 and 1286-57) were received directly from Michael Grimes (Department of Dairy and Food Microbiology, University College, Cork, Ireland), who identified them as Aerobacter liquefaciens. Strain 446-68 (= ATCC 
14460) was labeled Aerobacter lipolyticus by Grimes (16). Strain 1361-60 (L418) was received from the National Collection of Marine Bacteria and originally was identified as Aeromonas liquefaciens. This isolant was recovered from canal water in Delft by Soeters (see reference 6 and pages 5 and 31 of reference 13). The two remaining strains were received from the ATCC: $1159-70$ (=ATCC 25642) and 1160-70 (= ATCC 25643).

During this same period, 49 strains that resembled $B$. rubidaeum were received and examined. A few of these had been described as serratiae of one species or another and were supplied by the late R. S. Breed or by the ATCC: 2368-57 (= Breed CVIII, $S$. marcescens), 2375-57 (= Breed CU93, S. marcescens), 1209-70 (=ATCC 11634, S. marcescens), 1210-70 (=ATCC 15928, S. marcescens), and 1211-70 to 1214-70 (= ATCC 19278 to 19281, S. marinorubra). These eight strains were subsequently identified by the authors as $B$. rubidaeum. The two strains from R. S. Breed, CVIII and CU93, were isolated many years ago and were studied by numerous investigators, including Dr. Breed (personal communication, 1957) and the authors (11). The sources of the remaining isolants of $S$. liquefaciens and $B$. rubidaeum are given in Table 1 .

Methods. The methods used in the characterization of the strains were the same as those described elsewhere $(5,7,10,11,13,14)$. Unless otherwise

TABLE 1. Sources of cultures

\begin{tabular}{|c|c|c|}
\hline Source & $\begin{array}{c}\text { Serratia } \\
\text { liquefaciens }\end{array}$ & $\begin{array}{l}\text { Bacterium } \\
\text { rubidaeum }\end{array}$ \\
\hline \multicolumn{3}{|l|}{ Human } \\
\hline \multicolumn{3}{|l|}{ Extraintestinal } \\
\hline Blood ..... & 2 & 4 \\
\hline Ear . . . . . & 2 & \\
\hline Throat .... & 2 & 2 \\
\hline Sputum $\ldots . \ldots \ldots$ & $1 \overline{2}$ & 8 \\
\hline Lung $\ldots . . . . .$. & 2 & \\
\hline Empyema fluid .... & & 1 \\
\hline Wound, pus ...... & & 2 \\
\hline \multirow{2}{*}{\multicolumn{3}{|c|}{ Intestinal }} \\
\hline & & \\
\hline Bile $\ldots \ldots \ldots$ & 1 & 1 \\
\hline Feces .......... & 1 & \\
\hline Unknown $\ldots \ldots \ldots$ & 4 & 1 \\
\hline Animals $\ldots \ldots \ldots \ldots$ & 6 & $2^{b}$ \\
\hline Foods $^{a} \ldots \ldots \ldots \ldots$ & 51 & 1 \\
\hline \multicolumn{3}{|l|}{ Miscellaneous } \\
\hline Instruments . . . . . & 2 & \\
\hline Catheter lubricant ..... & & 1 \\
\hline Grass clippings $\ldots \ldots \ldots$ & 1 & \\
\hline Water $\ldots \ldots \ldots \ldots$ & 1 & 2 \\
\hline Culture collections ${ }^{c}$ & 6 & 8 \\
\hline Unknown $\ldots \ldots \ldots \ldots$ & 14 & $16^{d}$ \\
\hline Total ............ & 109 & 49 \\
\hline
\end{tabular}

${ }^{a}$ Includes 48 from dairy products.

${ }^{b}$ One from a bovine with mastitis.

${ }^{c}$ See text.

${ }^{d}$ Ten of these were received from Hans Lautrop of the State Serum Institute, Copenhagen, Denmark. indicated, the temperature of incubation was 35 to 37 C. Most of the isolants were tested repeatedly, but some of the older stock cultures were nonviable or could not be located. Hence, it was impossible to examine all isolates with all tests and substrates.

\section{RESULTS}

All of the strains studied contained gramnegative, rod-shaped cells which grew on ordinary media without enrichment and without increased carbon dioxide tension. Motile forms of $S$. liquefaciens and $B$. rubidaeum were peritrichous. The biochemical characteristics of the strains are presented below.

Serratia liquefaciens. Data resulting from the examination of the 109 strains of $S$. liquefaciens are summarized in Table 2. Two of the 19 isolates that fermented rhamnose also produced indole (Table 2). Since these strains were typical in other respects, they are regarded as biotypes of $S$. liquefaciens. The majority were typical and yielded reactions similar to those of strain number 1284-57 (Table 2), which is designated below as the type strain of $S$. liquefaciens. Most of the information given in Table 2 is self-explanatory, but a few comments may be helpful.

Some strains of $S$. liquefaciens gave negative Voges-Proskauer (VP) tests (Table 2), but there were no other significant differences between these isolants and their VP-positive counterparts. Therefore, the VP-negative form is regarded by the authors as a biotype of $S$. liquefaciens analogous to the VP-negative biotype of $S$. marcescens.

The strains that failed to decarboxylate lysine (Table 2) all gave positive ornithine reactions, and all those that failed to decarboxylate ornithine were lysine-positive. The single strain that deaminated phenylalanine (Table 2) was typical in other respects.

The isolates that failed to ferment sorbitol all produced acid from arabinose, and those that failed to utilize arabinose all fermented sorbitol (Table 2). Four of the five strains that failed to produce acid from raffinose were otherwise typical, but one also failed to ferment sorbitol (Table 2).

Two of the 19 isolants that produced acid from rhamnose failed to ferment sorbitol. One of these utilized both sodium mucate and sodium malonate, and another did not hydrolyze corn oil. Otherwise, the rhamnosepositive isolates were typical.

The strains that did not produce demonstrable extracellular deoxyribonuclease (DNase) 
TABLE 2. Summary of the biochemical reactions of Serratia liquefaciens, including the type strain

\begin{tabular}{|c|c|c|c|}
\hline Test or substrate & Reaction $^{\alpha}$ & $\begin{array}{c}\text { Percent }+ \\
\text { (109 strains) }\end{array}$ & Type strain $b$ \\
\hline Hydrogen sulfide $\ldots \ldots \ldots \ldots \ldots \ldots$ & - & 0 & - \\
\hline Urease $\ldots \ldots \ldots \ldots \ldots \ldots \ldots \ldots$ & $\mathrm{d}$ & $3.7^{\mathrm{w}}(11)^{c}$ & - \\
\hline Indole $\ldots \ldots \ldots \ldots \ldots \ldots \ldots$ & - & $1.8^{\mathrm{w}}$ & - \\
\hline Methyl red $(37 \mathrm{C}) \ldots \ldots \ldots \ldots \ldots$ & + or - & 64.2 & + \\
\hline Methyl red $(22 C) \ldots \ldots \ldots \ldots \ldots$ & - or + & 36.7 & + \\
\hline Voges-Proskauer $(37 \mathrm{C}) \ldots \ldots \ldots \ldots$ & - or + & 49.5 & + \\
\hline Voges-Proskauer $(22 \mathrm{C}) \ldots \ldots \ldots \ldots$ & + or - & 86.2 & + \\
\hline Citrate (Simmons) . . . . . . . . . . & + & $93.6(6.4)$ & + \\
\hline $\mathrm{KCN} \ldots \ldots \ldots \ldots \ldots \ldots \ldots \ldots \ldots \ldots$ & + & 91.7 & + \\
\hline Motility (peritrichous cells) . . . . . . . . . & + & 92.7 & $+($ see Fig. 1$)$ \\
\hline Gelatin $(22 \mathrm{C}) \ldots \ldots \ldots \ldots \ldots \ldots \ldots$ & + & $96.3(3.7)$ & + \\
\hline Lysine decarboxylase $(37 \mathrm{C}) \ldots \ldots \ldots \ldots$ & + or $(+)$ & $64.2(31.2)$ & + \\
\hline Lysine decarboxylase $(22 \mathrm{C}) \ldots \ldots \ldots \ldots$ & + & 96.3 & + \\
\hline Arginine dihydrolase $(37 \mathrm{C}) \ldots \ldots \ldots$ & - & 0 & - \\
\hline Arginine dihydrolase $(22 \mathrm{C}) \ldots \ldots \ldots$ & - & 0 & - \\
\hline Ornithine decarboxylase $(37 \mathrm{C}) \ldots \ldots \ldots$ & + & 100 & + \\
\hline Ornithine decarboxylase $(22 \mathrm{C}) \ldots \ldots \ldots$ & + & 99.1 & + \\
\hline Phenylalanine deaminase $\ldots \ldots \ldots \ldots \ldots$ & - & 0.9 & - \\
\hline Glucose acid $\ldots \ldots \ldots \ldots \ldots \ldots$ & + & 100 & + \\
\hline 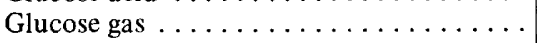 & $\mathrm{d}$ & $72.5(0.9)$ & + \\
\hline Lactose $\ldots \ldots \ldots \ldots \ldots \ldots \ldots$ & d & $15.6(21)$ & $\left(+_{9}\right)^{d}$ \\
\hline Sucrose $\ldots \ldots \ldots \ldots \ldots \ldots \ldots$ & + & 98.2 & + \\
\hline Mannitol $\ldots \ldots \ldots \ldots \ldots \ldots \ldots$ & + & 100 & + \\
\hline Dulcitol . . . . . . . . . . . . & - & 0 & - \\
\hline Salicin $\ldots \ldots \ldots \ldots \ldots \ldots \ldots$ & + & 96.3 & + \\
\hline Adonitol acid $\ldots \ldots \ldots \ldots \ldots \ldots$ & $\mathrm{d}$ & $8.3(5.5)$ & - \\
\hline Adonitol gas $\ldots \ldots \ldots \ldots \ldots \ldots \ldots$ & - & $0.9(1.8)$ & - \\
\hline Inositol acid $\ldots \ldots \ldots \ldots \ldots \ldots$ & + or $(+)$ & $64.2(25.7)$ & + \\
\hline Inositol gas $\ldots \ldots \ldots \ldots \ldots \ldots$ & $\mathrm{d}$ & $1.8(12.8)$ & + \\
\hline Sorbitol acid $\ldots \ldots \ldots \ldots \ldots \ldots$ & + & 97.3 & + \\
\hline Sorbitol gas $\ldots \ldots \ldots \ldots \ldots \ldots \ldots$ & $d$ & $57.8(19.3)$ & + \\
\hline Arabinose $\ldots \ldots \ldots \ldots \ldots \ldots$ & + & 97.3 & + \\
\hline Raffinose $\ldots \ldots \ldots \ldots \ldots \ldots \ldots$ & + & $90.8(4.6)$ & + \\
\hline Rhamnose $\ldots \ldots \ldots \ldots \ldots \ldots \ldots$ & $\mathrm{d}$ & $16.5(0.9)$ & -- \\
\hline  & - & 0.9 & - \\
\hline Mucate $\ldots \ldots \ldots \ldots \ldots \ldots \ldots$ & - & 0.9 & - \\
\hline Christensen citrate $\ldots \ldots \ldots \ldots \ldots$ & + & 95.2 & + \\
\hline Jordan tartrate $\ldots \ldots \ldots \ldots \ldots \ldots$ & + or - & 70.3 & - \\
\hline Sodium acetate $\ldots \ldots \ldots \ldots \ldots \ldots$ & d & $38.4(37.7)$ & - \\
\hline Sodium alginate $\ldots \ldots \ldots \ldots \ldots$ & - & 0 & - \\
\hline Pectate $\ldots \ldots \ldots \ldots \ldots \ldots \ldots$ & - & 0 & - \\
\hline Lipase, corn oil $\ldots \ldots \ldots \ldots \ldots \ldots$ & + or $(+)$ & $85.9(4.7)$ & + \\
\hline 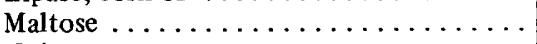 & + & $96.6(3.6)$ & + \\
\hline 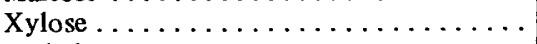 & + & $99.1(0.9)$ & + \\
\hline Trehalose $\ldots \ldots \ldots \ldots \ldots \ldots \ldots$ & + & 100 & + \\
\hline Cellobiose acid $\ldots \ldots \ldots \ldots \ldots \ldots$ & d & $27.3(36.3)$ & $\left(+_{8}\right)$ \\
\hline Cellobiose gas $\ldots \ldots \ldots \ldots \ldots \ldots$ & d & $7.1(32.3)$ & $\left(+_{8}\right)$ \\
\hline Glycerol acid $\ldots \ldots \ldots \ldots \ldots \ldots \ldots$ & + & $92.2(6.8)$ & + \\
\hline Glycerol gas $\ldots \ldots \ldots \ldots \ldots \ldots \ldots$ & d & $39.8(30.1)$ & + \\
\hline Alpha methyl glucoside $\ldots \ldots \ldots \ldots \ldots$ & - & $7.3(2.4)$ & - \\
\hline
\end{tabular}

${ }^{a}$ Symbols:,$+ 90 \%$ or more positive within 1 or 2 days of incubation; $(+)$, positive reaction after 3 or more days (decarboxylase reactions: 3 or 4 days); - , no reaction $(90 \%$ or more); + or - , most strains positive, some negative; - or + , most strains negative, some positive; + or $(+)$, most reactions occur within 1 or 2 days, some are delayed; $d$, different reactions $[+,(+),-]$. The percentages given in Table 2 are based on numerical data given in Table 4 of reference 9.

b Grimes no. 2 (1284-57 = ATCC 27592).

$c$ Numbers in parentheses indicate percentages of delayed reactions ( 3 or more days). Abbreviation: $w$, weak.

${ }^{d}$ Subscript numbers indicate day on which reaction occurred. 
TABLE 2-Continued

\begin{tabular}{|c|c|c|c|}
\hline Test or substrate & Reaction $^{a}$ & $\begin{array}{c}\text { Percent }+ \\
\text { (109 strains) }\end{array}$ & Type strain $b$ \\
\hline 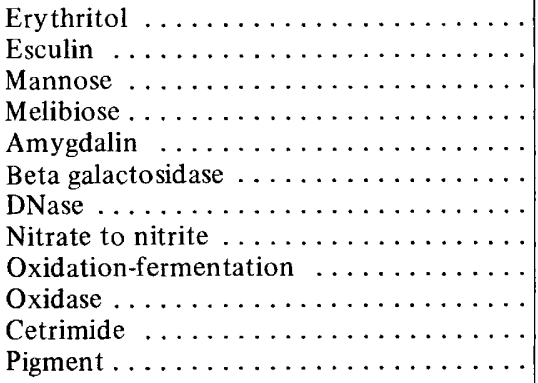 & $\begin{array}{l}- \\
+ \\
+ \\
\mathrm{d} \\
+ \\
+ \\
+ \text { or }- \\
+ \\
\text { F } \\
- \\
- \\
-\end{array}$ & $\begin{array}{l}0 \\
95.1(3.7) \\
100 \\
73.8(7.5) \\
100 \\
92.7 \\
88.3 \\
100 \\
100 \\
0 \\
0 \\
0\end{array}$ & $\begin{array}{l}- \\
+ \\
+ \\
+ \\
+ \\
+ \\
+ \\
+ \\
\text { F } \\
- \\
- \\
-\end{array}$ \\
\hline
\end{tabular}

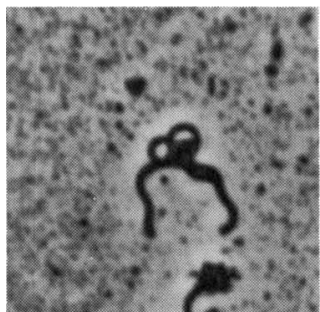

FIG. 1. Peritrichous cell of Serratia liquefaciens ATCC 27592 showing both coiled and normal flagella. Cells with normal flagella usually predominate. Twenty-four-hour broth culture at $26 \mathrm{C}$. Staining by Leifson's method. $\times 1,400$.

were all lipolytic (Table 2), and those that failed to hydrolyze corn oil produced extracellular DNase. Other aberrancies encountered (Table 2) were similar to those already described (14).

As mentioned previously $(14,16,17)$, cultures of $S$. liquefaciens tend to be more active metabolically at 22 to $25 \mathrm{C}$ than at 35 to $37 \mathrm{C}$. This tendency is apparent in the data presented in Table 2 (see also reference 14).

Supplementary tabular data and other details concerning the biochemical reactions of $S$. liquefaciens, and of $B$. rubidaeum (below), are given in a publication from the CDC (9), which is available upon receipt of a request addressed to one of the authors at the CDC.

Bacterium rubidaeum. The results obtained from the examination of 49 strains of $B$. rubidaeum are summarized in Table 3 . The delayed reactions recorded in this table occurred within 3 to 7 days of incubation.

Most of the isolates were typical and yielded reactions similar to those of strain 2199-72 (Table 3), which is designated below as the neotype of this species, but 18 strains were aberrant with respect to one or more of their reactions. Twelve of these deviatea in oniy one character, whereas six were aberrant in two or more of their reactions. The details of these aberrant characters are given in reference 9.

Three of the four strains labeled $S$. marinorubra when received from the ATCC failed to grow on Simmons citrate medium. These were regarded as slightly atypical isolants of $B$. rubidaeum. Another strain (1210-70 = ATCC 15928), labeled S. plymouthica when received, gave several aberrant reactions but was identified as an atypical strain of $B$. rubidaeum.

\section{DISCUSSION}

A comparison of the data on the biochemical reactions given by strains of $S$. liquefaciens (Tables 1-5) with those obtained with $S$. marcescens (8) indicates that $S$. liquefaciens is more closely related to Serratia than to any other genus in the family Enterobacteriaceae and that suggestions $(1,4,15,18)$ regarding its transfer to Serratia should be followed. Evidence of the relatedness of the deoxyribonucleic acids of $S$. marcescens and $S$. liquefaciens (3) confirms the desirability of the transfer. However, it is equally clear that the integrity of each of the species should be maintained; that is, $S$. liquefaciens should not be incorporated into $S$. marcescens. The data disclose at least five major differences between strains of these two species (boldface items in. Table 4). Other, less striking differences also are apparent (Table 4 and text). Moreover, Grimes regarded his $A$. liquefaciens as a cold-tolerant, mesophilic microorganism, and it is clear that these bacteria tend to be more active metabolically at 22 to $25 \mathrm{C}$ than at 35 to $37 \mathrm{C}$ 
TABLE 3. Summary of the biochemical reactions of Serratia rubidaea, including the neotype strain

\begin{tabular}{|c|c|c|c|}
\hline Test or substrate & Reaction $^{a}$ & $\begin{array}{c}\text { Percent }+ \\
\text { (49 strains) }\end{array}$ & Neotype strain $b$ \\
\hline Hydrogen sulfide $\ldots \ldots \ldots \ldots \ldots \ldots$ & - & 0 & - \\
\hline Urease $\ldots \ldots \ldots \ldots \ldots \ldots \ldots \ldots$ & $\mathrm{d}$ & $4^{\mathrm{w}}\left(16^{\mathrm{w}}\right)^{c}$ & - \\
\hline Indole $\ldots \ldots \ldots \ldots \ldots \ldots \ldots \ldots$ & - & $2^{w}$ & - \\
\hline Methyl red $(37 \mathrm{C}) \ldots \ldots \ldots \ldots \ldots$ & - or + & 31 & - \\
\hline Methyl red $(22 \mathrm{C}) \ldots \ldots \ldots \ldots \ldots \ldots$ & - or + & 24 & - \\
\hline Voges-Proskauer $(37 \mathrm{C}) \ldots \ldots \ldots \ldots$ & + & 92 & + \\
\hline Voges-Proskauer $(22 \mathrm{C}) \ldots \ldots \ldots \ldots$ & + & 94 & + \\
\hline Citrate (Simmons) $\ldots \ldots \ldots \ldots \ldots \ldots$ & + or $(+)$ & $88(2)$ & + \\
\hline 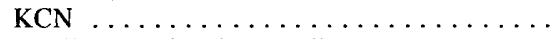 & - or + & 22 & - \\
\hline Motility (peritrichous cells) .......... & + or - & 88 & $+($ see Fig. 2$)$ \\
\hline Gelatin $(22 \mathrm{C}) \ldots \ldots \ldots \ldots \ldots \ldots$ & + or $(+)$ & $88(12)$ & + \\
\hline Lysine decarboxylase $(37 \mathrm{C}) \ldots \ldots \ldots \ldots$ & + or $(+)$ & $61(31)$ & + \\
\hline Lysine decarboxylase $(22 \mathrm{C}) \ldots \ldots \ldots \ldots$ & d & $45(6)$ & - \\
\hline Arginine dihydrolase $(37 \mathrm{C}) \ldots \ldots \ldots$ & - & 0 & - \\
\hline Arginine dihydrolase $(22 \mathrm{C}) \ldots \ldots \ldots$ & - & 0 & - \\
\hline Ornithine decarboxylase $(37 \mathrm{C}) \ldots \ldots \ldots$ & - & 0 & - \\
\hline Ornithine decarboxylase $(22 \mathrm{C}) \ldots \ldots \ldots$ & - & 0 & - \\
\hline Phenylalanine deaminase $\ldots \ldots \ldots \ldots \ldots$ & - & 0 & - \\
\hline Glucose acid $\ldots \ldots \ldots \ldots \ldots \ldots$ & + & 100 & + \\
\hline Glucose gas $\ldots \ldots \ldots \ldots \ldots \ldots$ & $\mathrm{d}$ & $35(4)$ & - \\
\hline Lactose $\ldots \ldots \ldots \ldots \ldots \ldots \ldots$ & + & 100 & + \\
\hline Sucrose $\ldots \ldots \ldots \ldots \ldots \ldots \ldots$ & + & 96 & + \\
\hline Mannitol $\ldots \ldots \ldots \ldots \ldots \ldots \ldots$ & + & 100 & + \\
\hline Dulcitol $\ldots \ldots \ldots \ldots \ldots \ldots \ldots$ & -. & 0 & - \\
\hline 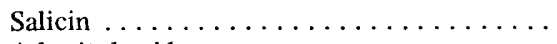 & + or $(+)$ & $88(4)$ & + \\
\hline Adonitol acid $\ldots \ldots \ldots \ldots \ldots \ldots$ & + or $(+)$ & $88(2)$ & + \\
\hline Adonitol gas $\ldots \ldots \ldots \ldots \ldots \ldots \ldots$ & - & 0 & - \\
\hline Inositol acid $\ldots \ldots \ldots \ldots \ldots \ldots \ldots$ & $\mathrm{d}$ & $35(16)$ & $\left(+_{3}\right)^{d}$ \\
\hline Inositol gas $\ldots \ldots \ldots \ldots \ldots \ldots \ldots$ & $\leftarrow$ & 0 & - \\
\hline Sorbitol acid $\ldots \ldots \ldots \ldots \ldots \ldots$ & - & 8 & - \\
\hline Sorbitol gas $\ldots \ldots \ldots \ldots \ldots \ldots$ & - & 0 & - \\
\hline Arabinose $\ldots \ldots \ldots \ldots \ldots \ldots \ldots$ & + & 100 & + \\
\hline Raffinose $\ldots \ldots \ldots \ldots \ldots \ldots$ & + & 96 & + \\
\hline Rhamnose $\ldots \ldots \ldots \ldots \ldots \ldots \ldots$ & - & $4(2)$ & - \\
\hline 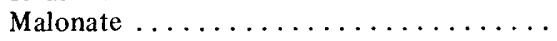 & + or - & 86 & + \\
\hline Mucate $\ldots \ldots \ldots \ldots \ldots \ldots \ldots \ldots$ & - & 0 & - \\
\hline Christensen citrate $\ldots \ldots \ldots \ldots \ldots \ldots$ & + & 100 & + \\
\hline Jordan tartrate $\ldots \ldots \ldots \ldots \ldots \ldots$ & + or - & 78 & + \\
\hline Sodium acetate $\ldots \ldots \ldots \ldots \ldots \ldots$ & d & $67(14)$ & - \\
\hline Pectate $\ldots \ldots \ldots \ldots \ldots \ldots \ldots \ldots$ & - & 0 & - \\
\hline Lipase, corn oil ............... & + & 98 & + \\
\hline Maltose $\ldots \ldots \ldots \ldots \ldots \ldots \ldots$ & + & 92 & + \\
\hline Xylose $\ldots \ldots \ldots \ldots \ldots \ldots \ldots \ldots$ & + & 98 & + \\
\hline 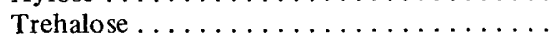 & + & 100 & + \\
\hline Cellobiose acid $\ldots \ldots \ldots \ldots \ldots \ldots$ & + & $90(6)$ & + \\
\hline Cellobiose gas $\ldots \ldots \ldots \ldots \ldots \ldots \ldots$ & - & 0 & - \\
\hline Glycerol acid $\ldots \ldots \ldots \ldots \ldots \ldots \ldots$ & $\mathrm{d}$ & $29(18)$ & + \\
\hline Glycerol gas $\ldots \ldots \ldots \ldots \ldots \ldots \ldots$ & - & 0 & - \\
\hline Alpha methyl glucoside $\ldots \ldots \ldots \ldots \ldots$ & $\mathrm{d}$ & $8(4)$ & - \\
\hline Erythritol $\ldots \ldots \ldots \ldots \ldots \ldots \ldots$ & - & $2(4)$ & - \\
\hline Esculin $\ldots \ldots \ldots \ldots \ldots \ldots \ldots \ldots$ & + & $90(8)$ & + \\
\hline 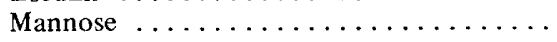 & + & 100 & + \\
\hline Melibiose $\ldots \ldots \ldots \ldots \ldots \ldots \ldots$ & + & 96 & + \\
\hline Amygdalin . . . . . . . . . . . . & + & 94 & + \\
\hline Beta galactosidase $\ldots \ldots \ldots \ldots \ldots \ldots$ & + & 100 & + \\
\hline 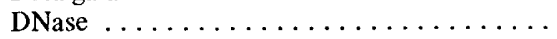 & + & 100 & + \\
\hline Nitrate to nitrite $\ldots \ldots \ldots \ldots \ldots \ldots$ & + & 100 & + \\
\hline Oxidation-fermentation ............. & $\mathrm{F}$ & 100 & $\mathrm{~F}$ \\
\hline Oxidase $\ldots \ldots \ldots \ldots \ldots \ldots$ & - & 0 & - \\
\hline Cetrimide $\ldots \ldots \ldots \ldots \ldots \ldots \ldots$ & $\mathrm{d}$ & $12(2)$ & - \\
\hline Pigment (pink to red) $\ldots \ldots \ldots \ldots \ldots$ & + or - & 61 & + \\
\hline
\end{tabular}

${ }^{a}$ See Table 2, footnote $a$, for explanation of symbols.

b Strain no. 2199-70 (= ATCC 27593).

${ }^{c}$ Numbers in parentheses indicate percentage of delayed reactions ( 3 days or more). Abbreviation: $w$, weak.

$a$ Subscript numbers indicate day on which reaction occurred. 
(Table 2 and reference 14). Cultures of $S$. marcescens and B. rubidaeum are not known to exhibit this tendency (Table 3 and references 8 , 14). Therefore, the authors support the transfer of E. liquefaciens to the genus Serratia as previously suggested by Bascomb et al. (1).

ATCC 14460 was deposited in the American Type Culture Collection by R. E. Buchanan, who received it from $M$. Grimes with the information that it was the type strain of Aerobacter lipolyticus (syn.: Aerobacter liquefaciens, Enterobacter liquefaciens, Serratia liquefaciens). This strain was cited as the type

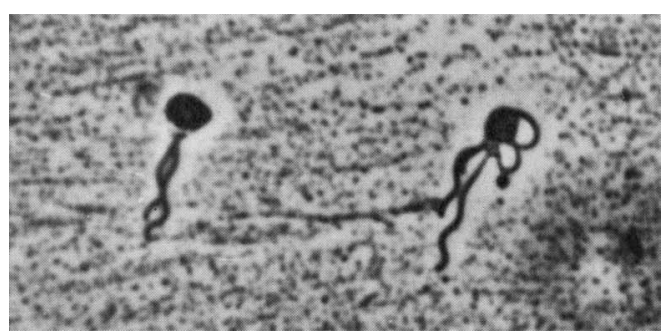

FIG. 2. Peritrichous cells of Serratia rubidaea ATCC 27593. The cell on the left has normal flagella and that on the right has both coiled and normal flagella. Cells with normal flagella predominate. Twenty-fourhour broth culture at $26 \mathrm{C}$. Staining by Leifson's method. $\times 1,400$. strain of Enterobacter liquefaciens in the seventh edition of the ATCC's Catalogue of Cultures (20). However, catalogues do not constitute effective publication insofar as nomenclatural matters are concerned, and to our knowledge a type strain never has been formally proposed for the microorganism of Grimes and Hennerty. Bascomb et al. (1, p. 293) made the following statement: "A type strain is not suggested [for Serratia liquefaciens (Grimes and Hennerty) Bascomb et al.] since the type strain for Enterobacter liquefaciens (ATCC 14460, NCIB 9321; Grimes \& Hennerty, 1931), was not included in this study." In this contradictory statement it is inferred that a type strain (ATCC 14460) exists for Enterobacter liquefaciens; presumably Bascomb et al. accepted the statement in the ATCC catalogue as establishing the type. Nevertheless, as mentioned above, the type strain for this microorganism heretofore has not been properly designated. Because strain 446-68 (= ATCC 14460 ) is somewhat atypical, it is proposed that strain 1284-57 (= ATCC 27592), which was received directly from Dr. Grimes and which has the characters of $A$. liquefaciens as originally reported by Grimes and Hennerty (17), be designated as the type strain of $S$. liquefaciens.

Similarly, it is apparent that the microorga-

TABLE 4. Differentiation of S. liquefaciens and $S$. marcescens ${ }^{a}$

\begin{tabular}{|c|c|c|c|c|}
\hline \multirow[b]{2}{*}{ Test or substrate } & \multicolumn{2}{|c|}{ S. liquefaciens } & \multicolumn{2}{|c|}{ S. marcescens } \\
\hline & Reaction $^{b}$ & $\begin{array}{c}\text { Percent }+ \\
\text { (109 strains })\end{array}$ & Reaction $^{b}$ & Percent + \\
\hline Urease $\ldots \ldots \ldots \ldots \ldots$ & $d$ & $3.7^{\mathrm{w}}\left(11^{\mathrm{w}}\right)^{\mathrm{c}}$ & $\mathrm{d}$ & $39.4^{\mathrm{w}}\left(22.3^{\mathrm{w}}\right)^{c}$ \\
\hline Methyl red $(37 \mathrm{C}) \ldots$ & + or - & 64.2 & - or + & 18.5 \\
\hline Methyl red $(22 \mathrm{C})$ & - or + & 36.7 & - & 8.8 \\
\hline Voges-Proskauer. & - or + & 49.5 & + & 98.7 \\
\hline Lysine decarboxylation ...... & + or $(+)$ & $64.2(31.2)$ & + & 99.6 \\
\hline Lactose . . . . . . . . . & $\mathrm{d}$ & $15.6(21)$ & - & $1.3(4.6)$ \\
\hline Adonitol $\ldots \ldots \ldots \ldots$ & d & $8.3(5.5)$ & $d$ & $46.5(13.8)$ \\
\hline Sorbitol gas & $\mathrm{d}$ & $57.8(19.3)$ & - & 0 \\
\hline Arabinose $\ldots \ldots \ldots \ldots \ldots$ & + & 97.3 & - & 0 \\
\hline Raffinose $\ldots \ldots \ldots \ldots$ & + & $90.8(4.6)$ & - & $1.2(0.8)$ \\
\hline Rhamnose ........ & $\mathrm{d}$ & $16.5(0.9)$ & - & 0 \\
\hline Xylose . . . . . . . . & + & $99.1(0.9)$ & $\mathrm{d}$ & $7.1(17.2)$ \\
\hline Cellobiose gas ..... & d & $7.1(32.3)$ & - & 0 \\
\hline Glycerol gas ...... & d & $39.8(30.1)$ & - & 0 \\
\hline Ery thritol $\ldots \ldots \ldots \ldots \ldots$ & - & 0 & $\mathrm{~d}$ & $8(25.1)$ \\
\hline Melibiose . . . . . . . . & $d$ & $73.8(7.5)$ & - & 0 \\
\hline
\end{tabular}

${ }^{a}$ Based on data in Table 2 and references 8 and 9.

b See Table 2, footnote $a$, for explanation of symbols.

$c$ Numbers in parentheses indicate percentages of delayed reactions ( 3 days or more). Abbreviation: w, weak.

${ }^{d}$ Boldface indicates substrates of particular differential value. 
nism called $B$. rubidaeum (22) is closely related to $S$. marcescens and $S$. liquefaciens (Tables 2 and 3 ; reference 9). Strains of $B$. rubidaeum appear to be intermediate between those of $S$. marcescens and $S$. liquefaciens. They share many characteristics with each of these species
(Tables 2-6; reference 8) but are sufficiently different from either (Tables 5,6 ) so as to be regarded as a separate species. The characters in boldface type in Tables 4-6 indicate the principal differences between the three species; other important differences are also apparent in

TABLE 5. Differentiation of S. liquefaciens and S. rubidaea ${ }^{a}$

\begin{tabular}{|c|c|c|c|c|}
\hline \multirow[b]{2}{*}{ Test or substrate } & \multicolumn{2}{|c|}{ S. liquefaciens } & \multicolumn{2}{|c|}{ S. rubidaea } \\
\hline & Reaction $^{b}$ & $\begin{array}{c}\text { Percent }+ \\
\text { (109 strains) }\end{array}$ & Reaction $^{b}$ & $\begin{array}{c}\text { Percent }+ \\
\text { (49 strains) }\end{array}$ \\
\hline Voges-Proskauer .. & - or + & 49.5 & + & 92 \\
\hline $\mathrm{KCN} \ldots \ldots \ldots \ldots \ldots$ & + & 91.7 & - or + & 22 \\
\hline Ornithine decarboxylase $^{c} \ldots \ldots \ldots$ & + & 100 & - & 0 \\
\hline Lactose . . . . . . . . . . & d & $15.6(21)^{d}$ & + & 100 \\
\hline Adonitol $\ldots \ldots \ldots \ldots \ldots$ & $\mathrm{d}$ & $8.3(5.5)$ & + or $(+)$ & $88(2)$ \\
\hline Sorbitol acid . . . . . . & + & 97.3 & - & 8 \\
\hline Sorbitol gas . . . . . . . . & $\mathrm{d}$ & $57.8(19.3)$ & - & 0 \\
\hline Rhamnose $\ldots \ldots \ldots \ldots \ldots$ & $\mathrm{d}$ & $16.5(0.9)$ & - & $4(2)$ \\
\hline Malonate $\ldots \ldots \ldots \ldots \ldots \ldots$ & - & 0.9 & + or - & 86 \\
\hline Cellobiose acid $\ldots \ldots \ldots \ldots \ldots$ & $\mathrm{d}$ & $27.3(36.3)$ & + & $90(6)$ \\
\hline Cellobiose gas ........ & $\mathrm{d}$ & $7.1(32.3)$ & - & 0 \\
\hline Glycerol acid & + & $92.2(6.8)$ & $\mathrm{d}$ & $29(18)$ \\
\hline Glycerol gas ........ & $\mathrm{d}$ & $39.8(30.1)$ & - & 0 \\
\hline
\end{tabular}

${ }^{a}$ Based on data in Tables 2 and 3.

$b$ See footnote $a$, Table 2, for explanation of symbols.

$c$ Boldface indicates substrates of particular differential value.

$d$ Numbers in parentheses indicate percentages of delayed reactions ( 3 days or more). Delayed reactions given by $S$. rubidaea occurred within 3 to 7 days.

TABLE 6. Differentiation of S. rubidaea and S. marcescens ${ }^{a}$

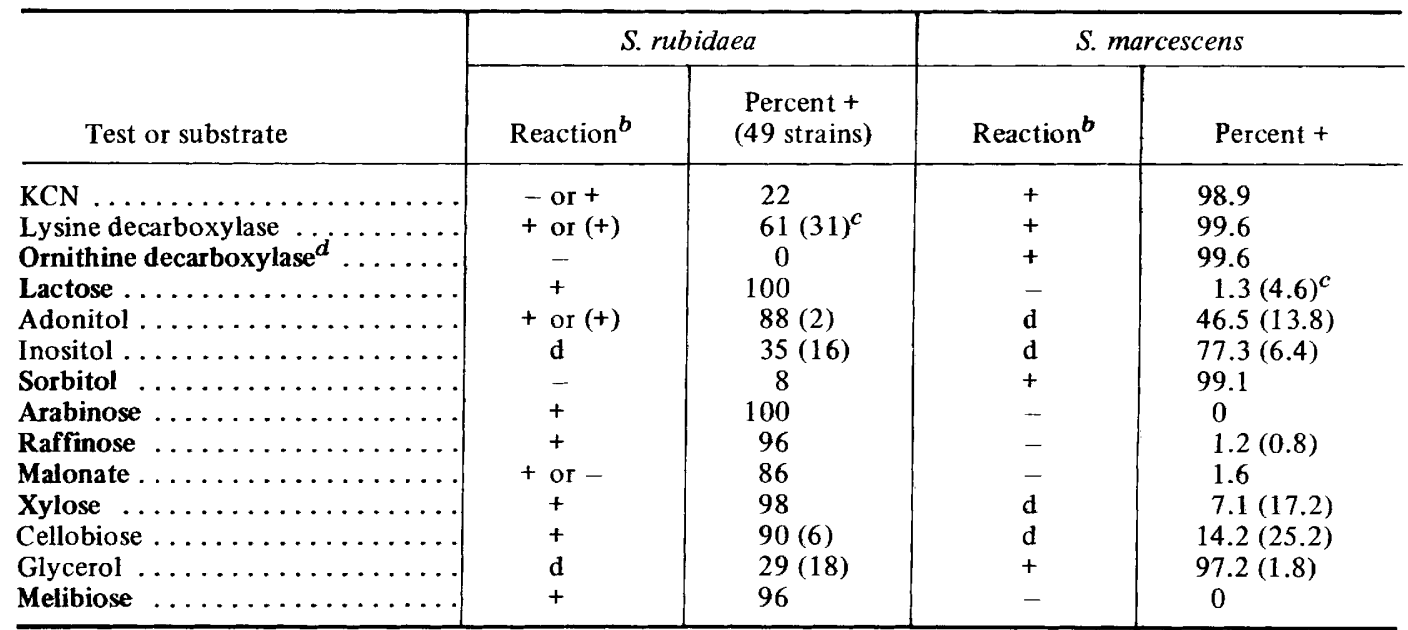

${ }^{a}$ Based on data in Table 3 and references 8 and 9.

$b$ See footnote $a$, Table 2, for explanation of symbols.

${ }^{c}$ Numbers in parentheses indicate percentages of delayed reactions ( 3 days or more). Delayed reactions given by $S$. rubidaea occurred within 3 to 7 days.

$d$ Boldface indicates substrates of particular differential value. 
the tables. With few exceptions, isolants of these three species can be differentiated with ease in the authors' experience. In addition to the substrates listed in Tables 4 and 5 , it has been found that gas production from esculin also is of assistance in the differentiation of $S$. liquefaciens from $S$. marcescens and $B$. rubidaeum (14). Many strains of $S$. liquefaciens produce gas from esculin, whereas members of the other species are not known to do so (14). Similarly, the volumes of gas formed from fermentable substrates ( 10 to $25 \%$ or more) by aerogenic isolates of $S$. liquefaciens are, in general, larger than those produced by $S$. marcescens or B. rubidaeum. Rapid fermentation of lactose by all strains of $B$. rubidaeum examined (Tables 3, 5, 6) also is helpful for delineation of members of this species. However, strains of $B$. rubidaeum that ferment lactose slowly or that do not produce acid from lactose may be isolated in the future.

Therefore, the authors propose that $B$. rubidaeum Stapp be transferred to the genus Serratia and be recognized as the third species in this genus. Stapp (22) has been cited (2) as the author of the name Serratia rubidaea. However, when he proposed the name Bacterium rubidaeum, Stapp stated: "Nach Bergey's Manual müsste er, sofern der Gattungsname 'Serratia' internationale Anerkennung findet, 'Serratia rubidaea' heissen müssen." Thus Stapp merely noted that, if the generic name Serratia finds international acceplance, according to Bergey's Manual the name Bacterium rubidaeum would have to be "Serratia rubidaea." According to Rule $12 \mathrm{c}(1)$ and (2) of the International Code of Nomenclature of Bacteria (19), S. rubidaea was not validly published by Stapp, and so Serratia rubidaea is proposed here as a new combination. Strain $2199-72$ (= ATCC 27593) is designated as the neotype strain of $S$. rubidaea. The characters of this strain agree very well with those recorded in the original description of $B$. rubidaeum by Stapp (22). One difference noted is that Stapp reported fermentation of sorbitol; the neotype strain does not produce acid from sorbitol, but neither do $92 \%$ of the strains of $S$. rubidaea studied here.

The cultures of $S$. marinorubra ZoBell and Upham 1944 received from the ATCC are indistinguishable from $S$. rubidaea.

According to the proposals made, the nomenclature of the three species recognized in the genus Serratia is as follows. Genus Serratia Bizio: (i) Serratia marcescens Bizio; (ii) Serratia liquefaciens (Grimes and Hennerty) Bascomb et al.; (iii) Serratia rubidaea (Stapp) comb. nov.

\section{REPRINT REQUESTS}

Address requests for reprints to: Dr. William H. Ewing, U.S. Department of Health, Education and Welfare, Center for Discase Control, Atlanta, Ga. 30333 .

\section{LITERATURE CITED}

1. Bascomb, S., S. P. Lapage, W. R. Willcox, and M. A. Curtis. 1971. Numerical classification of the tribe Klebsielleae. J. Gen. Microbiol. 66:279-295.

2. Breed, R. S., F. G. D. Murray, and A. P. Hitchens. 1948. Bergey's manual of determinative bacteriology, 6th ed. The Williams \& Wilkins Co., Baltimore.

3. Brenner, D. J., A. G. Steigerwalt, and G. R. Fanning. 1972. Differentiation of Enterobacter aerogenes from Klebsiellae by deoxyribonucleic acid reassociation. Int. J. Syst. Bacteriol. 22:193-200.

4. Durand, A. M., and D. J. Blazevic. 1970. Differentiation of Serratia from Enterobacter on the basis of nucleoside phosphotransferase production. Appl. Microbiol. 19:134-137.

5. Edwards, P. R., and W. H. Ewing. 1972. Identification of Enterobacteriaceae, 3rd ed. Burgess Publishing Co., Minneapolis.

6. Ewing, W. H. 1963. An outline of nomenclature for the family Enterobacteriaceae. Int. Bull. Bacteriol. Nomencl. Taxon. 13:95-110.

7. Ewing, W. H., and B. R. Davis. 1970. Media and tests for differentiation of Enterobacteriaceae. Center for Disease Control, Atlanta.

8. Ewing, W. H., and B. R. Davis. 1972. Biochemical characterization of Serratia marcescens. Publ. Health Lab. 30:211-226.

9. Ewing, W. H., B. R. Davis, and M. A. Fife. 1972. Biochemical characterization of Serratia liquefaciens and Serratia rubidaea. Center for Disease Control, Atlanta.

10. Ewing, W. H., B. R. Davis, and J. G. Johnson. 1962. The genus Serratia: its taxonomy and nomenclature. Int. Bull. Bacteriol. Nomencl. Taxon. 12:47-52.

11. Ewing, W. H., B. R. Davis, and R. W. Reavis. 1959. Studies on the Serratia group. Center for Disease Control, Atlanta.

12. Ewing, W. H., and P. R. Edwards. 1960. The principal divisions and groups of Enterobacteriaceae. Int. Bull. Bacteriol. Nomencl. Taxon. 10:1-12.

13. Ewing, W. H., R. Hugh, and J. G. Johnson. 1961. Studies on the Aeromonas group. Center for Disease Control, Atlanta.

14. Fife, M. A., W. H. Ewing, and B. R. Davis. 1965. The biochemical reactions of the tribe Klebsielleae. Center for Disease Control, Atlanta.

15. Greenup, P., and D. J. Blazevic. 1971. Antibiotic susceptibilities of Serratia marcescens and Enterobacter liquefaciens. Appl. Microbiol. 22:309-314.

16. Grimes, M. 1961. Classification of the KlebsiellaAerobacter group with special reference to the 
cold-tolerant mesophilic Aerobacter types. Int. Bull. Bacteriol. Nomencl. Taxon. 11:111-129.

17. Grimes, M., and A. J. Hennerty. 1931. A study of bacteria belonging to the sub-genus Aerobacter. Sci. Proc. Royal Dublin Soc. 20(NS):89-97.

18. Hamon, Y., L. LeMinor, and Y. Peron. 1970. Les bactériocines d'Enterobacter liquefaciens. C.R. Acad. Sci. 270:886-889.

19. International Code of Nomenclature of Bacteria. 1966. Int. J. Syst. Bacteriol. 16:459-490.
20. Lessel, E. F. (ed.). 1964. American Type Culture Collection Catalogue of Cultures. 7 th edition. American Type Culture Collection, Rockville, Maryland.

21. Monreal, J., and E. T. Reese. 1969. The chitinase of Serratia marcescens. Can. J. Microbiol. 15:689-696.

22. Stapp, C. 1940. Bacterium rubidaeum nov. $s p$. Zentralbl. Bakteriol. Il Abt. 102:251-260. 